\title{
An under-actuated origami gripper with adjustable stiffness joints for multiple grasp modes
}

\author{
Amir Firouzeh and Jamie Paik \\ Reconfigurable Robotics Lab, EPFL, Lausanne, Switzerland. \\ E-mail: jamie.paik@epfl.ch
}

December 2016

\begin{abstract}
Under-actuated robots offer multiple degrees of freedom without much added complexity to the actuation and control. Utilizing adjustable stiffness joints in these robots allows us to control their stable configurations and their mode of interaction with the environment. In this paper, we present the design of tendon-driven robotic origami (robogami) joints with adjustable stiffness. The proposed designs allow us to place joints along any direction in the plane of the robot and in the normal direction to the plane. The layer-by-layer manufacturing of robogamis facilitates the design and manufacturing of robots with different arrangement of joints for different applications.

We use thermally activated shape memory polymer to control the joint stiffness. The manufacturing of the polymer layer is compatible with the layer-by-layer manufacturing process of the robogamis which results in scalable and customizable robots. To demonstrate, we prototyped an under-actuated gripper with three fingers and only one input actuation. The grasp mode of the gripper is set by adjusting the configuration of the locked joints and modulating the stiffness of the active joints. We present a model to estimate the configuration and the contact forces of the gripper at different settings that will assist us in design and control of future generation of under-actuated robogamis.
\end{abstract}

\section{Introduction}

Safe and adaptable interaction with the environment requires operational fluidity. This can be achieved in robots with multiple and kinematically redundant degrees of freedom (DoFs). At the same time, the quest for miniaturization in emerging applications for mobile robots necessitates simplicity and compactness of the actuation mechanism in the robots. Driving several DoF with a single actuation source in under-actuated robots results in systems that are easier to actuate and control compared to fully actuated robots [1-6]. Moreover, under-actuated systems have the advantage of inherent softness which is a desired feature in many applications where safe interaction with a sensitive environment is necessary. We aim to enhance the performance of the under-actuated robots by integrating a part of the control in the structure of the robot using materials 
with adjustable stiffness. This method enables us to control the stable configuration and the force exerted on the environment by an under-actuated system.

Robotic origamis (robogami) are low profile robots that use the layer-by-layer manufacturing process [7] for integrating layers with different functions. Accurate quasi2D fabrication methods for preparing each of the layers makes the overall structure accurate and highly scalable. In this paper, we introduce tendon-driven robotic origami joints for DoFs along any desired direction in the plane of the robot and also along the normal to the plane of the robot. The axes of rotation for the joints in the plane of the robot are determined by the folding pattern of a polyimide sheet, which is imposed on it by castellated patterns of glass fiber layers $[8,9]$. For the joint in the normal direction, the axis of rotation is defined by the rolling contact of the two tiles. Once the joint design is finalized, any arrangement of the joints for fulfilling a specific task can be designed and manufactured without any added complexity.

We embed adjustable stiffness layers as one of the functional layers in the robogamis to control the configuration of a robot with multiple DoFs and a single source of actuation. Many methods were proposed for controlling the stiffness of the segments and joints for achieving compliant actuation in robots ranging from methods that use mechanical systems [10-13] to methods that rely on the adjustable material properties [14-29]. Among these, stiffness control through utilizing the glass transition in polymers is a choice compatible with the layer-by-layer manufacturing process of the robogamis. Among the thermoplastic polymers, shape memory polymers (SMPs) have the added advantage of better shape recovery at temperatures higher than their glass transition. The temperature of the SMP layer, hence its stiffness, is controlled using a stretchable metal mesh heater which is embedded inside SMP [14]. The embedded heater provides uniform heating power in the SMP layer and allows us to control the stiffness of the joints in increments.

The main contribution of this work is the introduction of the tendon-driven robogami joints with adjustable stiffness layer (ASL) that allows us to arrange DoFs along any desired axis in the plane of the robot and along the normal to the plane. Any arrangement of these joints can be designed for covering a desired workspace. We utilize the proposed design for the joints in an under-actuated gripper to activate different grasp modes presented in Fig. 1. The gripper reconfigures from its initial planar state into different 3D configurations. The layer-by-layer construction along with precise nominally 2D machining processes enables us to design structures with multiple DoFs and varied possible configurations for handling objects of different shapes and sizes. The gripper prototype confirms the feasibility of the joint designs, the manufacturing process, and the under-actuation of multiple joints using a singles source. To best utilize the adaptive stiffness of the robogami joints and its design versatility, we propose a model for the tendon-driven robogamis which will assist us in design of the future generations of under-actuated robogamis. We study the kinetostatic model of the fingers in the gripper and based on this, we propose a control scheme for effectively using the workspace of the fingers and the adjustable stiffness of the joints for grasping objects with different 
shapes. The implementation of this control scheme requires further studies. In this paper, we demonstrate grasping objects in few basic modes.

In Section 2, we introduce a three-fingered robogami gripper which is used as a case study to confirm the feasibility of the joint design and manufacturing process. In Section 3, we present the design of the robogami joints and their working principle and estimate the joint stiffness based on the stiffness of the ASL. In Section 4, we describe the layer-by-layer manufacturing process, the design, and the fabrication process of the ASL. Finally in Section 5, the kinetostatic model of the fingers of the gripper is presented which will assist us in designing and controlling the future generations of under-actuated robogamis for robotic grippers and other applications.

\section{Three-fingered robogami gripper with adjustable grasp modes}

One of the applications that can benefit from adjustable stiffness joints is the underactuated robotic grippers. Stiffness control of the joints allows us to adjust the operation mode of the gripper based on the required transformations and contact forces. As a case

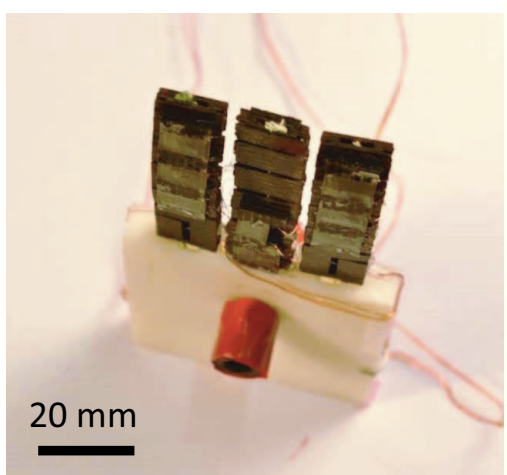

(a)
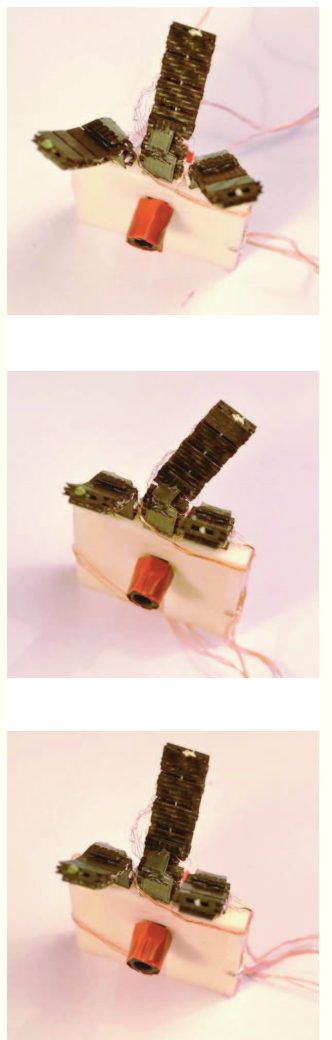

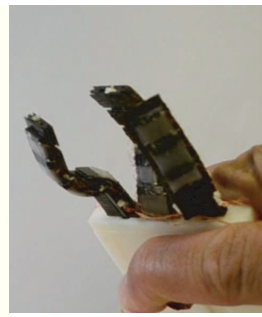

(b)

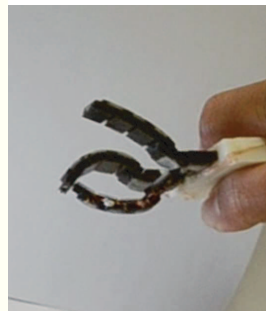

(c)

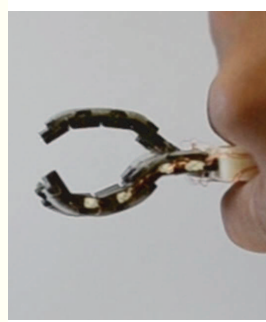

(d)
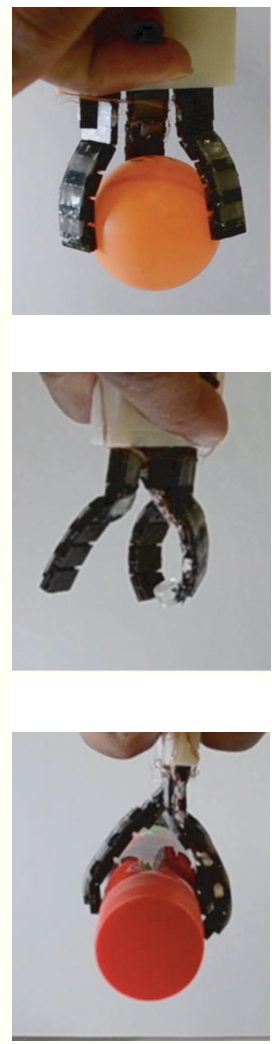

Figure 1: An under actuated gripper with adjustable stiffness joint and multiple grasp modes. (a) The three fingers start from completely flat state: initial state. (b) The cylindrical grasp mode : all active joints have parallel axes of rotation. (c) The spherical grasp mode : the side fingers are oriented according to the shape of the object for better grasp. (d) The two finger pinching grasp : the middle finger is rotated to face one of the side fingers for handling small objects. 
study we consider a three-fingered gripper with multiple grasp modes as presented in Fig. 1. This manually actuated gripper showcases the applicability of the robogami joints with adjustable stiffness and the under-actuation of multiple joints using a single actuation source.

The gripper was designed to allow multiple modes of operation based on the initial configuration and active joints. Fig. 2 presents the schematic of the actuation system and the joint sequences in the three fingers of the gripper in the present design. The input sliding motion drives four tendons using a seesaw and pulley mechanism. Neglecting the friction forces, the tension is expected to be the same in all tendons and equal to the quarter of the input force. The deformation of each joint depends on the stiffness assigned to that joint and all the rest of the joints.

The first two joints in each finger is used for changing the initial configuration of the gripper from the flat state, Fig. 1a, for different grasp modes: cylindrical grasp (Fig. 1b), spherical grasp (Fig. 1c), and two-finger pinching grasp (Fig. 1d). In each finger, the first y axis joint has the opposite actuation direction compared to all the rest of the $y$ axis joints in that finger. This allows the fingers to start from entirely flat state as presented in Fig. 1a and transform to the cylindrical grasping mode that is presented in Fig. 1b. For the two side fingers we added a rotational DoF along the $\mathrm{x}$ axis. This allows us to orient these fingers for grasping objects with different shapes, as presented in Fig. 1c. For the middle finger we have added a DoF along the $\mathrm{z}$ axis. Using this DoF

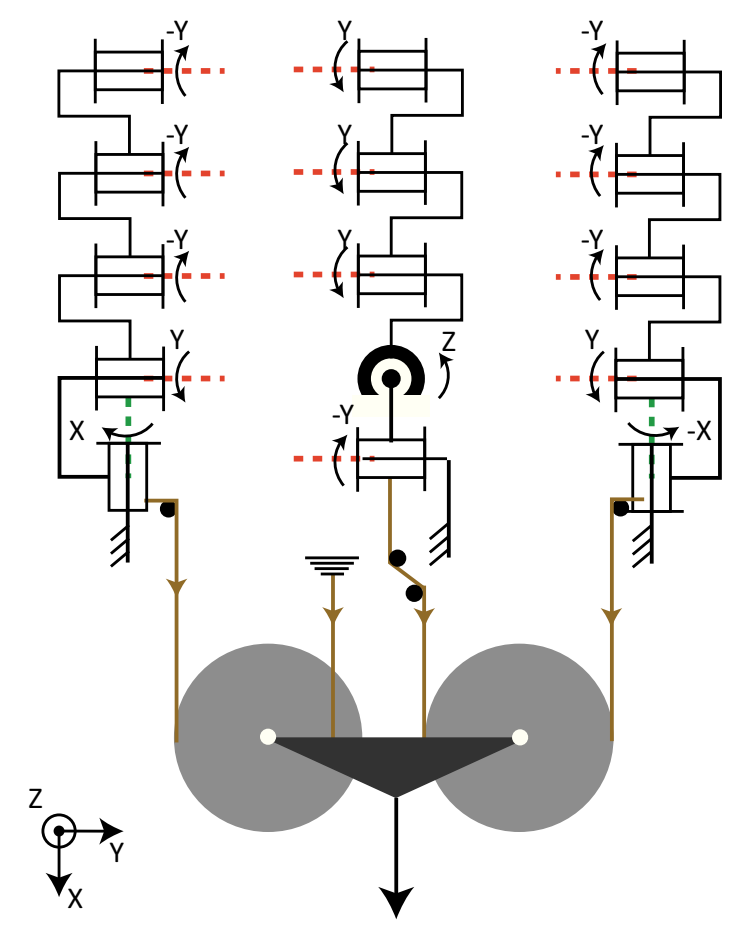

Figure 2: The schematic of the actuation mechanism and the DoFs of each finger. The input force is divided through the seesaw mechanism and the pulleys between the three finger. Neglecting the friction, the tendon tension in each finger equals to a quarter of the input force. The displacement of the tendons in each finger will be dependent on the joint stiffness assignment. 
we can move the tip of the middle finger to face one of the side fingers as presented in Fig. 1d. This mode of operation is preferable for handling small objects using only two fingers.

\section{Robogami joints with adjustable stiffness}

The gripper fingers are based on the robogami design, which relies on the layer-by-layer manufacturing process. This fabrication process allows us to design and manufacture robots with many DoFs in different directions. In this Section, the joint designs for rotation along the three orthogonal axes are presented. Each finger of the gripper is made by a series of the joints that are introduced here. The overview of the different joints is presented in Table 1. The joint designs for the $\mathrm{x}$ and $\mathrm{y}$ axes are similar and only the tendon path is different for the two designs. In these joints, the castellated pattern of the glass fiber layers determines the axis of rotation and the range of motion. For the rotation along the $\mathrm{z}$ axis, the joint design is different. In this design, the rolling

Table 1: The design of the three joints for DoFs along the three orthogonal axes. The polyimide layer and the castellated pattern on the glass fiber layer defines the axes of rotation for DoFs along $\mathrm{x}$ and $\mathrm{y}$. For the $\mathrm{z}$ axis joints the design is different and the rolling motion of the two adjacent tiles define the axis of rotation. The top layer is made transparent to depict the tendon routing in the three joints.

\begin{tabular}{|l|l|l|l|}
\hline $\begin{array}{l}\text { Axis } \\
\text { of ro- } \\
\text { tation }\end{array}$ & Initial configuration & Joint displacement & $\begin{array}{l}\text { View along the axis of } \\
\text { rotation }\end{array}$ \\
\hline y-axis & & &
\end{tabular}


contact point between the two tiles defines the axis of rotation and the ASL confines the relative motion of the two tiles. The tendon is routed on the opposite side of the ASL to apply the required moment for the deformation. The top layer in the schematics of Table 1 are made transparent to highlight the path of the tendon in the tiles. Changing the direction of the force through guiding the tendon in a desired direction allows us to drive robots with joints along the three orthogonal axes using a single tendon for actuation. The deformation of the ASL layer is also depicted in the schematics of Table 1. Controlling the stiffness of ASLs allows us to control the compliance of each joint and hence the actuation angle for a given tendon force.

To estimate the joint stiffness, we study the deformation of the ASL layer in different joints. The schematic of the joints along different axes depicting the joint motion and the design parameters are presented in Fig. 3. For the joints along the $\mathrm{x}$ and $\mathrm{y}$ axes, the strain along the width of the ASL is constant. The required moment for rotating these joint is calculates as:

$$
M_{x, y}\left(\theta_{0}, \delta \theta, T\right)=F_{A S L}\left(\theta_{0}, \delta \theta, T\right) h \cos (\theta / 2)
$$

In (2), $F_{A S L}$ and $M_{x, y}$ are the required force in ASL and the bending moment for reaching a desired bending angle, $\theta$. Differentiating (1), the stiffness for these joints, $k_{x, y}$, is calculated as follows:

$$
k_{x, y}=\frac{\partial M_{x, y}}{\partial \theta}=\frac{1}{l_{A S L}} \frac{\partial F_{A S L}}{\partial l_{A S L} / l_{A S L}} \frac{\partial l_{A S L}}{\partial \theta} h \cos (\theta / 2)-\frac{F_{A S L}}{2} h \sin (\theta / 2)
$$

In (2), $\frac{\partial F_{A S L}}{\partial l_{A S L} l_{A S L}}$ represents the stiffness of the ASL. This stiffness is a function of the temperature of the ASL. In the next section the construction of the ASL and

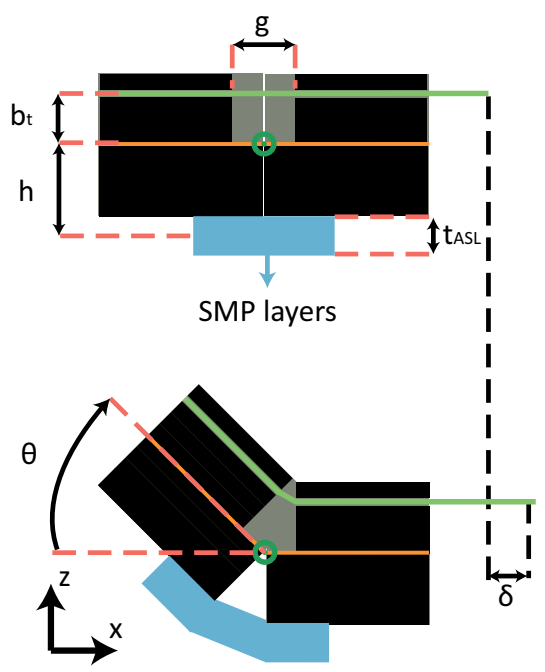

(a)

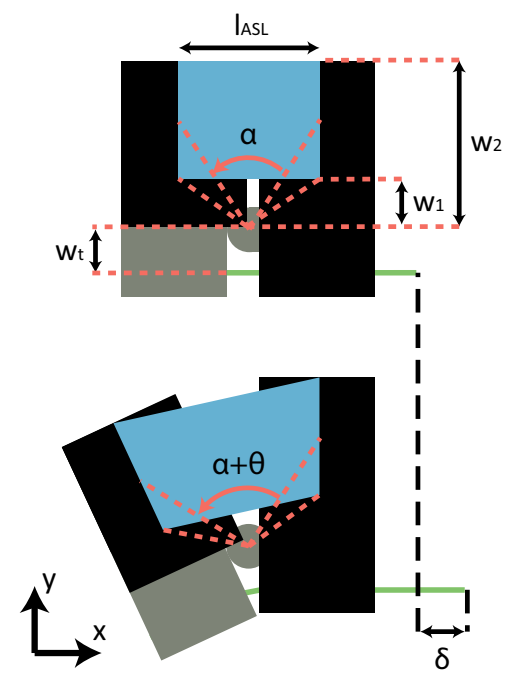

(b)

Figure 3: The schematic of the joints depicting deformation of the ASL and the tendon. (a) Joints along $\mathrm{x}$ and $\mathrm{y}$ axes. (b) Joints along $\mathrm{z}$ axis. 
its elastic behavior with temperature will be presented. For small angles around the straight configuration, $\theta=0$, the joint stiffness can be approximated as:

$$
k_{x, y}=\frac{k_{A S L} w_{A S L-x, y}}{l_{A S L}} h^{2}
$$

(3) $k_{A S L}$ represents the stiffness per unit width of the ASL and $w_{A S L-x, y}$ is the width of ASL for $\mathrm{x}$ and $\mathrm{y}$ joints.

For the $\mathrm{z}$ axis joints, the instantaneous axis of rotation is the contact point between the two tiles. The moving axis of rotation complicates the calculation of the joint stiffness for this case. Given the small radius of the rolling, $1.5 \mathrm{~mm}$, and the small goal angles around this joint, $25^{\circ}$ at maximum, we can simplify the problem by estimating the motion of this joint by a rotation along the fixed axis which passes through the center of the cylindrical part. Given the non-uniform strain along the width of the ASL, we have the following relation for the required moment for rotating the joint for small angles:

$$
M_{z}\left(\theta_{0}, \delta \theta, T\right)=\int_{w_{1}}^{w_{2}} k_{A S L}\left(\frac{y \sin \left(\frac{\theta}{2}\right)}{\frac{l_{A S L}}{2}}\right) \cos (\theta / 2) y \mathrm{~d} y
$$

For small angles around the straight configuration, the joint stiffness along the $\mathrm{z}$ axis is calculated as:

$$
k_{z}=k_{A S L} \frac{w_{2}^{3}-w_{1}^{3}}{3 l_{A S L}}
$$

Using (3) and (5) the joints along different axis can be designed to cover a desired stiffness range. In the prototype of Fig. 1 the ASL for $\mathrm{z}$ axis joint was designed to have the same stiffness as the joints along the other two axes. The design parameters for the joints are summarized in Table 2 .

Any sequence of the joints introduced in this Section can be designed to cover a desired workspace in an under-actuated robot. In the next Section, we introduce

Table 2: The values of the design parameters.

\begin{tabular}{|c|c|c|}
\hline Parameters & Value $(\mathrm{mm})$ & Description \\
\hline$l$ & 8.8 & Tile length \\
\hline$g$ & 1.4 & Gap size between the tiles \\
\hline$w_{A S L-x, y}$ & 7 & ASL width for the $\mathrm{x}$ and $\mathrm{y}$ joints \\
\hline$w_{A S L-z}$ & 4.4 & ASL width for the $\mathrm{z}$ joints \\
\hline$w_{1}$ & 0.6 & ASL distance from the $\mathrm{z}$ axis \\
\hline$w_{t}$ & 2.6 & Tendon distance from the $\mathrm{z}$ axis \\
\hline$l_{A S L}$ & 4 & ASL length in the active part \\
\hline$t_{A S L}$ & 1.6 & ASL thickness \\
\hline$h$ & 2.4 & ASL distance from the $\mathrm{x}$ and $\mathrm{y}$ axis \\
\hline$b$ & 1.1 & Tendon distance from the $\mathrm{x}$ and $\mathrm{y}$ axis \\
\hline
\end{tabular}


the layer-by-layer manufacturing process which is well adapted for the fabrication of the ASLs, the joints, and the tendon channels that are introduced in this Section. In Section 5, we present a model for the tendon-driven robogami and a control scheme which highlights the future approach for effectively designing and controlling robogamis capable of desired transformations and force application.

\section{Layer-by-layer manufacturing and examples of ASL elasticity variations with temperature}

Integrating different layers for manufacturing the structure of the joints requires novel fabrication processes. In this research, we adopted the layer-by-layer manufacturing process of the robogamis for each finger of the gripper. In this process, different functional layers are manufactured using accurate quasi-2D fabrication processes. Then these layers are bonded together to make the final robot. In this Section, first we present the manufacturing process of the ASLs and report on its elastic properties at different temperatures and then the overall design of the robogami and different layers of the structure are presented.

To control the stiffness of the structure, we use the variation of the elasticity modulus of a SMP (MM5520 from SMP Technologies changes), 2.1 GPa at room temperature to $2.1 \mathrm{MPa}$ at temperatures higher than its glass transition temperature

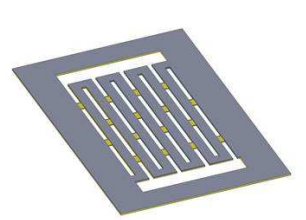

(a)

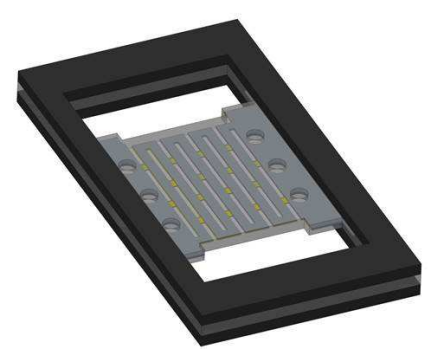

(c)

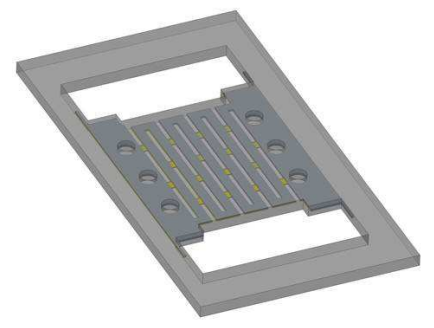

(b)

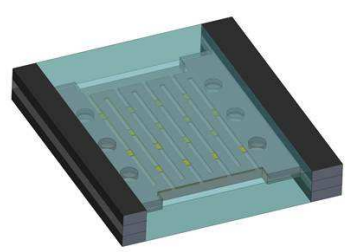

(d)

Figure 4: The manufacturing process of the ASL. (a) The stretchable heater is fabricated using a combination of laser micro-machining and chemical etching, refer to [30] for fabrication details. (b) The heater is embedded in SMP and its outline is cut using the laser. (c) The SMP layer is embedded in a glass fiber frame which acts as anchoring points for attaching to the structure and also as the mold for casting the silicone. (d) The SMP layer is encapsulated inside silicone rubber which enhances its shape recovery. 
(at $55^{\circ} \mathrm{C}$ ). To control the temperature of the SMP a stretchable heater (Fig. 4a) is embedded inside the SMP layer. The schematic of the heater in Fig. 4a is not to scale and the mesh structure is much finer than depicted (each column is $150 \mu \mathrm{m}$ wide). The outer frame of the heater is designed to prevent its elongation during the integration process in the SMP and as presented in Fig. 4b it is cut to release the heater in the second step. The shape memory effect of the SMP layer is desired and in the absence of external load, it drives the ASL to its initial shape. To improve the shape recovery, we encapsulated the SMP layer in silicone rubber, Dragon skin-30 from smooth-on. To enhance the mechanical bonding between the SMP and the silicone layer, holes are cut in the SMP layer as presented in Fig. 4b. The glass-fiber frame which is added to the SMP layer in the third step, Fig. 4c, makes the mold for the silicone rubber and also the anchoring point to connect the ASL to the robogami structure. Fig. 4d presents the ASL after cutting its outer frame.

The temperature of the SMP layer, hence the stiffness of the ASL, is controlled using a customized stretchable heater which is embedded in the SMP layer. Here, we used the electrical resistance of the heater to estimate and control the temperature, more details are presented in [21]. To verify the feasibility of the stiffness control, we characterized the elastic behavior of an ASL sample similar to the ones used in $\mathrm{x}$ and $\mathrm{y}$ axis joints at different temperatures. Fig. 5 presents the characterization test results for the ASL sample at different temperatures, from $30{ }^{\circ} \mathrm{C}$ to $110{ }^{\circ} \mathrm{C}$ in $10{ }^{\circ} \mathrm{C}$ increments. The sample at each temperature is stretched up to $9 \mathrm{~N}$ or $50 \%$ strain, whichever occurs first. The loading rate is $0.05 \mathrm{~mm} / \mathrm{s}$ till $50{ }^{\circ} \mathrm{C}$ and $0.3 \mathrm{~mm} / \mathrm{s}$ at higher temperatures. The deformation is applied using a spindle drive from Maxon motors with $10 \mu \mathrm{m}$ accuracy. The force is measured with a Nano 17 force sensor with

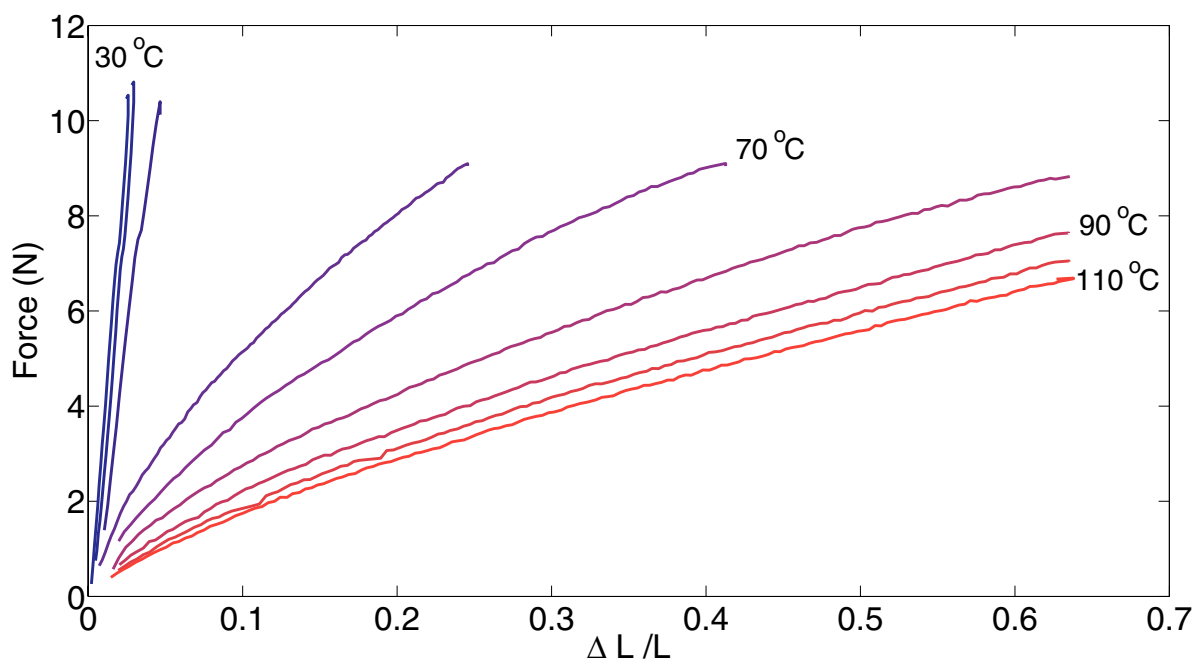

Figure 5: The ASL stiffness characterization at different temperature settings from $30{ }^{\circ} \mathrm{C}$ to $110{ }^{\circ} \mathrm{C}$ in $10{ }^{\circ} \mathrm{C}$ steps. The sample at each temperature is stretched up to $9 \mathrm{~N}$ or $50 \%$ strain, whichever occurs first. The thermal expansion, the memory effect of the SMP and the residual strain in the SMP layer result in the small variation in the initial point for different tests. The dimensions of the ASL sample $\left(w \times l \times t_{\text {total }}, t_{S M P}\right)$ are: $7 \times 4 \times 1.6,0.5 \mathrm{~mm}$. 
$3 \mathrm{mN}$ accuracy, more details about the test setup are presented in [18]. The overshoots in the force at lower temperatures and in strain at higher temperatures are due to the linear acceleration limits of the test setup which result in stretching the sample beyond the set points. The thickness of the heater and the SMP layer in the ASL are $100 \mu \mathrm{m}$ and $500 \mu \mathrm{m}$, respectively. The characterization results presented in Fig. 5 are for the ASL sample similar to the ASLs used in $\mathrm{x}$ and $\mathrm{y}$ axis joints. We estimate the behavior of the ASL with a linear function and use the slope as its stiffness $\left(k_{A S L}(T) w_{A S L-x, y}\right)$. The stiffness of the ASL layer changes more than 40 times depending on its temperature. This allows us to effectively control the stiffness of the robogami joints.

Fig. 6a presents how different layers are bonded to fabricate three joints of a robotic

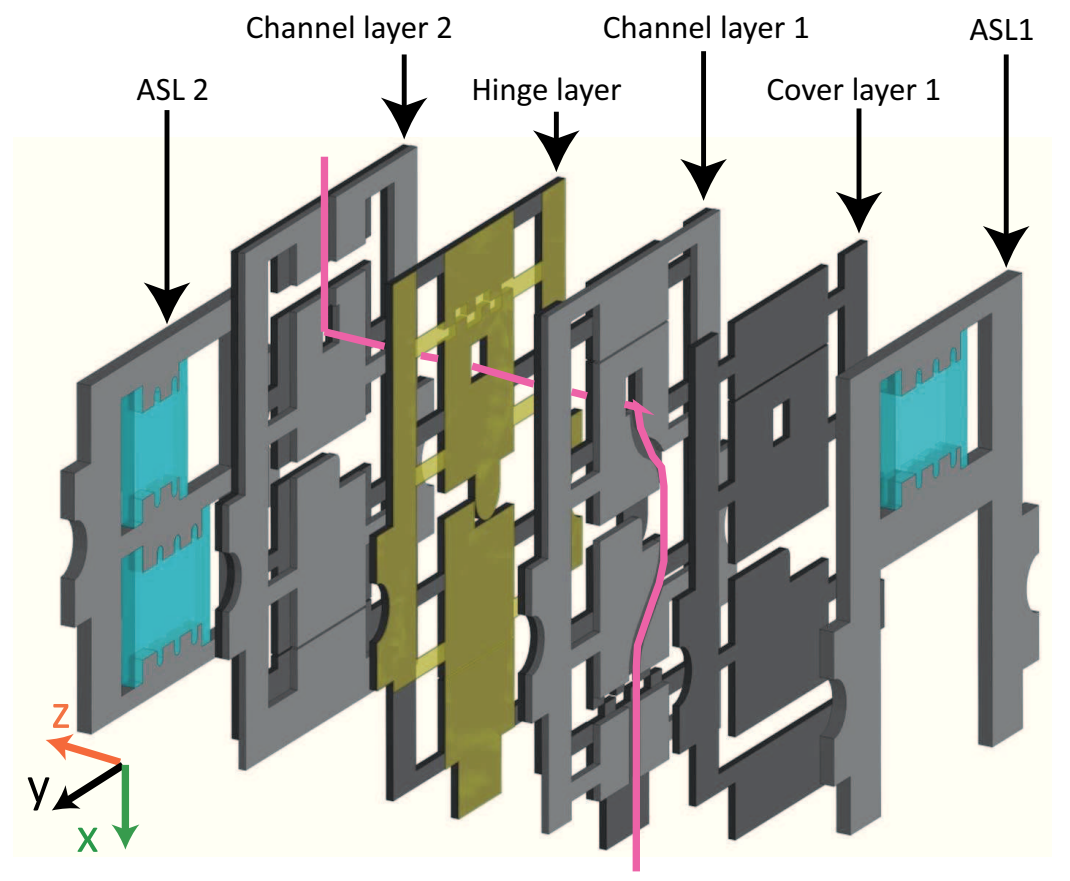

(a)

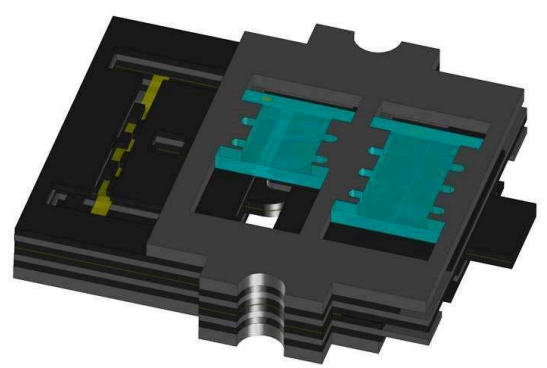

(b)

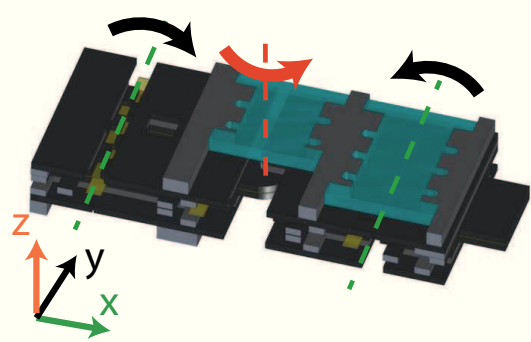

(c)

Figure 6: The layer-by-layer manufacturing process of a sequence of three joints. (a) Different layers are processed using accurate quasi-2D fabrication processes. These layers are aligned and bonded together using thermoset adhesive (pyralux LF0110, Dupont). The purple line in this figure shows the path of the the tendon. (b) The layers are bonded together to from the final structure. (c) The alignment structure which keeps the joints fixed during the bonding process is cut using laser to free the joints and finally the tendon is passed through the channel. 
finger. The first and the third joints in this design are both along the y axis but they have different directions of motion. The tendon channel is designed to direct the tendon from one side of the hinge layer to the other side which makes it possible to actuate the first and third joints in opposite directions. The middle joint in this design is along

the $\mathrm{z}$ axis. To actuate this joint, the glass fiber channel directs the tendon to apply the force at a desired distance from the axis of rotation. Since the ASL is more effective in tension, the ASL for the first and the third joints are placed on the opposite sides of the hinge layer. For the middle joint, the ASL is placed on the opposite side of the tendon channel to be on the tension side, Fig. 6 .

The structure of the joints is made of $500 \mu \mathrm{m}$ thick glass fiber layers. The cured glass fiber layers are patterned using a UV laser micro-machining station (details are presented in [31]). These layers along with the polyimide hinge layer are aligned in a jig and are bonded together in a heat press using thermoset adhesive layers (Pyralux LF0110). Fig. 6b presents the structure after the bonding step and Fig. 6c presents the final finger after the alignment structure is cut out. Finally the tendon is passed through the channel and is fixed on one end of the finger. The path of the tendon is highlighted in Fig. 6a which depicts how the design of different layers directs the tendon from one side of the hinge layer to the other side.

The layer-by-layer manufacturing process introduced in this Section can be adopted to fabricate structures with multiple joints with no added assembly time and with high accuracy. The design can easily be scaled down to include more DoF in a confined space. It can also be scaled up to increase the output force and the overall stiffness of the finger. Once the design of each joint is fixed, the patterns for different layers of any sequence of joints can easily be made by simply duplicating the pattern of a single joint. We use this manufacturing technique to fabricate the three fingers of the gripper presented in Fig. 1.

\section{Three-fingered gripper model and control scheme}

In the proposed design for the three-fingered gripper, the stiffness of the joints and the state of the locked joints depend on the desired motion from each finger. Here, we propose an algorithm to determine the initial configuration of the gripper and the stiffness of each active joint depending on the contact points on the object and the desired contact forces. The goal here is to highlight the approach to effectively utilize the DoFs of each finger and the adjustable stiffness of the joints for grasping objects with different sizes and shapes. The model and the control scheme, which are presented in this Section, provide an overview of the important design criteria which will assist us in designing the future generations of under-actuated robogamis for grippers and other applications.

Depending on the stiffness setting for the joints, the under-actuated gripper has some degree of self adaptability and exact control over the stiffness or positioning of the gripper base would not be necessary for performing the grasp motion. However, the 


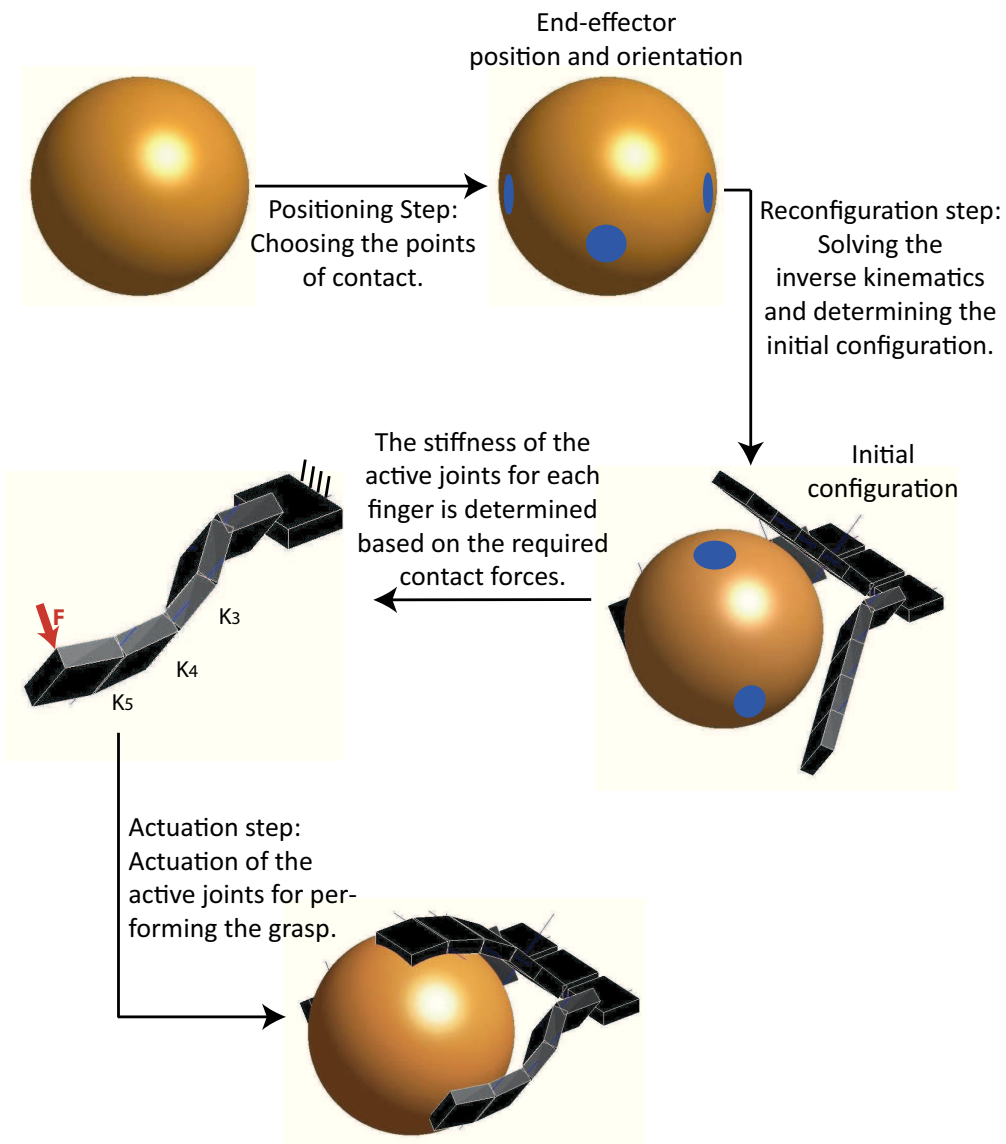

Figure 7: The overview of the control scheme for the gripper. Based on the shape of the object, the points of contact are chosen and the feasibility of the grasp is confirmed in an iterative process. Based on the position and the orientation of the contact points the inverse kinematics for each finger is solved. Finally, based on the desired contact force and the configuration of the finger the stiffness of each joint is assigned by solving the equilibrium equations for each finger.

study of the inverse kinematics and stiffness setting for the joints through kinetostatic model for each finger provides the general framework for setting the initial configuration of the gripper and the joints' stiffnesses.

The overview of the grasping algorithm is presented in Fig. 7. Here, we consider only one point of contact for each finger. In an iterative process we find the relative position for the object and the gripper in which we can choose points of contact on the surface that yield feasible configurations for the fingers. To simplify the problem, we considered only positions of the object along a fixed axis, step 1 in Fig. 7. The study of the equilibrium of the grasped object and formulating the complete grasping problem including the stability and robustness of the grasp according to the choice of contact points, orientation for each finger, and the stiffness of the joints is a topic for the future research.

The forward kinematics for the position and orientation of the last link of each 
finger are presented in (6) and (7).

$$
\begin{aligned}
& {\left[\begin{array}{l}
p \\
1
\end{array}\right]=e^{\hat{\xi_{1}} \theta_{1}} e^{\hat{\xi_{2}} \theta_{2}} e^{\hat{\xi_{3}} \theta_{3}} e^{\hat{\xi_{4}} \theta_{4}} e^{\hat{\xi_{5}} \theta_{5}}\left[\begin{array}{c}
p_{0} \\
1
\end{array}\right]} \\
& {\left[\begin{array}{c}
n \\
0
\end{array}\right]=e^{\hat{\xi_{1}} \theta_{1}} e^{\hat{\xi_{2}} \theta_{2}} e^{\hat{\xi_{3}} \theta_{3}} e^{\hat{\xi_{4}} \theta_{4}} e^{\hat{\xi_{5}} \theta_{5}}\left[\begin{array}{c}
n_{0} \\
0
\end{array}\right]}
\end{aligned}
$$

In (6) and (7), $\hat{\xi}_{i}$ is the $4 \times 4$ matrix representation of the twist corresponding to each joint axis and $\theta_{i}$ represents the joint angle. $e^{\hat{\xi}_{i} \theta_{i}}$ calculates the homogeneous transformation corresponding to the motion of each joint. In (6), $p_{0}$ and $p$ are the initial and final positions of the contact point on the last phalanx. In (7), $n_{0}$ and $n$ represents the initial and final normal to the last phalanx which corresponds to the direction of the force applied to the object. For a given position and orientation, we numerically solve these equations to find the appropriate configuration for each finger. Since there is the possibility of multiple solutions, other optimization goals, such as equal joint angle for all active joints, can be considered to choose the best among the possible solutions.

The first two joints in each finger are utilized for reconfiguration of the gripper from its flat state into the initial grasp states presented in Fig. 1. We refer to this step as reconfiguration step as presented in Fig. 7. The last three joints in each finger are the active joints that perform the grasping as presented in the actuation step in Fig. 7. The input displacement from the tendon is divided between these joints depending on their stiffness setting. The joints' stiffnesses are determined according to the configuration and the desired contact force for each finger using the kinetostatic model for the fingers.

$$
\mathbf{F}_{\mathbf{t}}^{T} \dot{\mathbf{X}}=W_{\text {contact }}+W_{A S L}
$$

In (8), the left side of the equation represents the input work. The elements of $\mathbf{F}_{\mathbf{t}}$ are the tension in the tendon at each joint and the elements of $\dot{\mathbf{X}}$ are the rate of the tendon displacement caused by the motion of each joint. We do not include the friction between the tendon and its channel in the model. So all the elements of $\mathbf{F}_{\mathbf{t}}$ are considered to be equal to the input tendon tension. The first term in the right side of this equation, $W_{\text {contact }}$, corresponds to the work done by the contact forces and the second term , $W_{A S L}$, correspond to the work done to deform the ASLs. As mentioned at this step we just consider one contact point at the last phalanx. Neglecting any friction at the contact point the contact work is calculated as:

$$
W_{\text {contact }}=f\left[\begin{array}{lll}
d_{35} & d_{45} & d_{55}
\end{array}\right] \dot{\theta}
$$

where $f$ is the contact forces, $d_{i 5}$ is the distance of the $i^{\text {th }}$ joint from the contact force vector of the last phalanx, and $\dot{\theta}$ is the vector of the joints' rotational speed.

The rate of the tendon displacement, $\dot{\mathbf{X}}$ in (8), has the following relation with the angular velocities of the joints:

$$
\dot{\mathbf{X}}=\mathbf{T} \dot{\theta}=\left[\begin{array}{ccc}
\frac{\partial X_{3}}{\partial \theta_{3}} & 0 & 0 \\
0 & \frac{\partial X_{4}}{\partial \theta_{4}} & 0 \\
0 & 0 & \frac{\partial X_{5}}{\partial \theta_{5}}
\end{array}\right] \dot{\theta}
$$


$\frac{\partial X_{i}}{\partial \theta_{i}}$ in this equation is the transmission ratio between the input and the $i^{\text {th }}$ joint. (11) represents the correlation between the rate of the joint angle change and the tendon displacement.

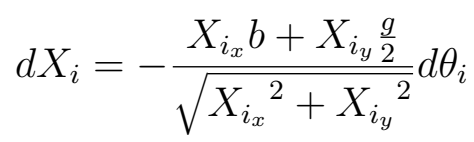

$X_{i_{x}}$ and $X_{i_{y}}$ are solved as:

$$
\begin{aligned}
& X_{i_{x}}=\frac{g}{2}\left(1+\cos \theta_{i}\right)-b \sin \theta_{i} \\
& X_{i_{y}}=\frac{g}{2}\left(\sin \theta_{i}\right)+b \cos \theta_{i}-b
\end{aligned}
$$

The rate of the energy storage in the ASL, $W_{A S L}$, is found as:

$$
W_{A S L}=\mathbf{F}_{\mathbf{A S L}^{T}}{ }^{T} \dot{\boldsymbol{\Delta}}=\left(\left[\begin{array}{ccc}
k_{3} & 0 & 0 \\
0 & k_{4} & 0 \\
0 & 0 & k_{5}
\end{array}\right] \boldsymbol{\Delta}\right)^{T} \dot{\boldsymbol{\Delta}}
$$

In (14), $\boldsymbol{\Delta}$ and $\mathbf{F}_{\mathbf{A S L}}$ represent the elongation and the force of the ASLs. $k_{i}$ represent the stiffness of the ASL layer of the corresponding joint. The ASL elongation, $\boldsymbol{\Delta}$, is a function of the joint angle and is calculated as:

$$
\Delta=2\left(h+\frac{t_{A S L}}{2}\right) \sin \frac{\theta}{2}
$$

Replacing the stored energy in the ALSs in (8) yields the following set of equations that correlate the tension in the tendon with the joint angles and their stiffness:

$$
\mathbf{F}_{\mathbf{t}}^{T} \mathbf{T}=\mathbf{f}^{T} \mathbf{J}+\left(h+\frac{t_{A S L}}{2}\right)^{2}\left(\mathbf{K}_{\mathbf{A S L}} \sin \theta\right)^{T}
$$

For a desired configuration and contact force, this set of equations determines the joint stiffness and the tendon displacement. Since there is one more variable than the equations, the solution is not unique. Considering the same tendon tension for all the fingers, we choose the answer that gives the minimum joint stiffness for the softest joint among all the fingers.

Using the kinematics model for the gripper and the kinetostatic model for each finger, the initial configuration and the stiffness of active joints can be determined for grasping different objects. To effectively apply the algorithm presented in this Section, further study of the stability of the grasp would be necessary. Moreover, a detailed model considering the tendon friction forces is necessary to closely simulate the behavior of the gripper's fingers for determining the stiffness of active joints.

As mentioned, using the model for determining the initial configuration and the stiffness of active joints requires more detailed model, motorizing the gripper, and incorporating sensors, which remain as topics for the future research. In the experiments on the gripper, we confirmed the applicability of the joint designs and feasibility of actuation of multiple fingers using a single source by performing few basic grasp modes that utilize different DoFs in each finger. 


\section{Conclusion}

In this paper we presented a novel design of tendon-driven robogami joints with adjustable stiffness. The proposed designs for the joints enable us to place DoFs along any direction on the plane of these low profile robots and also along the normal to the plane. Through controlling the stiffness of the under-actuated robogami joints, we can achieve different actuation patterns and modes of interaction with the environment.

To control the joint stiffness, we integrated a SMP layer in the joint area. The joint stiffness is controlled using the adjustable stiffness of the SMP layer around its glass transition point. To control the temperature of the SMP an stretchable heater was embedded in it and to increase the shape recovery rate, the SMP layer was embedded inside a silicone rubber layer. The ASL manufacturing process is compatible with the layer-by-layer manufacturing of the robogamis and it can easily be integrated in the robogami joints.

We used the joint designs in a gripper that is composed of three fingers that are driven with a single actuation source. A combination of a seesaw mechanism and pulleys divide the input motion between the fingers. We designed the fingers to achieve three major modes of operation: 1- cylindrical grasp, in which all the active joints are parallel; 2- spherical grasp, in which the side fingers rotate around the axes along their length to better orient themselves with contact points on the object; 3- pinching mode, in which the middle finger rotates along the axis normal to its plane to face its tip with one of the side fingers for grasping small objects.

We presented a model that relates the stable configuration and the contact forces to the joint stiffness. Based on this model we proposed a control scheme for finding the desired state of the locked joints and the stiffness of the active joints based on the object shape and size. To implement this control scheme further studies on the stability of the grasp, more detailed model considering the friction of the tendon, and sensors for estimating the configuration and contact forces are needed. In this work, we focused on and demonstrated the overall feasibility of the design and presented the result of successful grasp in the three major modes. Using the model presented here robogamis capable of desired transformations and covering a desired range of compliance variation can be designed for different applications.

\section{References}

[1] L. Birglen, T. Laliberte, and C. Gosselin, Underactuated Robotic Hands. (Springer Tracts in Advanced Robotics). Springer, 208, vol. 40.

[2] A. M. Dollar and R. D. Howe, "The highly adaptive sdm hand: Design and performance evaluation," The Int. J. Robot. Res., vol. 29, no. 5, pp. 585-597, 2010.

[3] D. M. Aukes, B. Heyneman, J. Ulmen, H. Stuart, M. R. Cutkosky, S. Kim, P. Garcia, and A. Edsinger, "Design and testing of a selectively compliant underactuated hand," The Int. J. Robot. Res., 2014.

[4] M. Ciocarlie, F. M. Hicks, R. Holmberg, J. Hawke, M. Schlicht, J. Gee, S. Stanford, and 
R. Bahadur, "The velo gripper: A versatile single-actuator design for enveloping, parallel and fingertip grasps," The Int. J. Robot. Res., vol. 33, no. 5, pp. 753-767, 2014.

[5] P. Qi, C. Qiu, H. Liu, J. S. Dai, L. D. Seneviratne, and K. Althoefer, "A novel continuum manipulator design using serially connected double-layer planar springs," IEEE/ASME TMech., vol. 21, no. 3, pp. 1281-1292, June 2016.

[6] W. Wang, H. Rodrigue, H.-I. Kim, M.-W. Han, and S.-H. Ahn, "Soft composite hinge actuator and application to compliant robotic gripper," Composites Part B, vol. 98, pp. 397 - 405, 2016.

[7] R. J. Wood, S. Avadhanula, R. Sahai, E. Steltz, and R. S. Fearing, "Microrobot design using fiber reinforced composites," J. Mech. Design, vol. 130, no. 5, pp. 052 304-052 304, 2008, $10.1115 / 1.2885509$.

[8] N. Doshi, B. Goldberg, R. Sahai, N. Jafferis, D. Aukes, R. J. Wood, and J. A. Paulson, "Model driven design for flexure-based microrobots," pp. 4119-4126, Sept 2015.

[9] M. T. Tolley, S. M. Felton, S. Miyashita, D. Aukes, D. Rus, and R. J. Wood, "Self-folding origami: shape memory composites activated by uniform heating," Smart Materials and Structures, vol. 23, no. 9, p. 094006, 2014. [Online]. Available: http://stacks.iop.org/0964$1726 / 23 / \mathrm{i}=9 / \mathrm{a}=094006$

[10] A. Degani, H. Choset, A. Wolf, and M. A. Zenati, "Highly articulated robotic probe for minimally invasive surgery," in IEEE Int. Conf. Robot. and Autom., pp. 4167-4172.

[11] J. Choi, S. Hong, W. Lee, S. Kang, and M. Kim, "A robot joint with variable stiffness using leaf springs," IEEE Tran. Robot., vol. 27, no. 2, pp. 229-238, April 2011.

[12] S. Wolf, G. Grioli, O. Eiberger, W. Friedl, M. Grebenstein, H. Hppner, E. Burdet, D. G. Caldwell, R. Carloni, M. G. Catalano, D. Lefeber, S. Stramigioli, N. Tsagarakis, M. V. Damme, R. V. Ham, B. Vanderborght, L. C. Visser, A. Bicchi, and A. Albu-Schffer, "Variable stiffness actuators: Review on design and components," IEEE/ASME T-Mech., vol. 21, no. 5, pp. 2418-2430, Oct 2016.

[13] R. A. Stavenuiter, L. Birglen, and J. L. Herder, "A planar underactuated grasper with adjustable compliance," Mech. and Mach. Theory, pp. -, 2016.

[14] A. Firouzeh, S. S. Mirrazavi Salehian, A. Billard, and J. Paik, "An under actuated robotic arm with adjustable stiffness shape memory polymer joints," in Proc. IEEE Int. Conf. Robot. and Autom., May 2015, pp. 2536-2543.

[15] M. A. McEvoy and N. Correll, "Thermoplastic variable stiffness composites with embedded, networked sensing, actuation, and control," J. Comp. Mat., 2014.

[16] W. Shan, T. Lu, and C. Majidi, "Soft-matter composites with electrically tunable elastic rigidity," Smart Mater. Struct., vol. 22, no. 8, p. 085005, 2013.

[17] L. Hines, V. Arabagi, and M. Sitti, "Shape memory polymer-based flexure stiffness control in a miniature flapping-wing robot," IEEE Tran. Robot., vol. 28, no. 4, pp. 987-990, 2012.

[18] A. Firouzeh, M. Salerno, and J. Paik, "Soft pneumatic actuator with adjustable stiffness layers for multi-dof actuation," in Proc. IEEE/RSJ Int. Conf. Int. Robot. and Sys., Sept 2015, pp. $1117-1124$.

[19] T. Ranzani, M. Cianchetti, G. Gerboni, I. D. Falco, and A. Menciassi, "A soft modular manipulator for minimally invasive surgery: Design and characterization of a single module," IEEE Tran. Robot., vol. 32, no. 1, pp. 187-200, Feb 2016.

[20] A. A. Stanley and A. M. Okamura, "Controllable surface haptics via particle jamming and pneumatics," IEEE Trans. Haptics, vol. 8, no. 1, pp. 20-30, Jan 2015.

[21] A. Firouzeh and J. k. Paik, "Stiffness control in under-actuated robotic origamis with shape memory polymer," in IEEE Transaction on Robotics, 2017, p. submitted Sep. 2016.

[22] J. Manzo and E. Garcia, "Analysis and optimization of the active rigidity joint," Smart Mater. Struct., vol. 18, no. 12, p. 125020, 2009.

[23] W. Ying, C. Yonghua, R. Tao, C. Qiao, Y. Changxin, Y. Yang, and L. Yingtian, "A novel, variable stiffness robotic gripper based on integrated soft actuating and particle jamming," Soft Robot., vol. 3(3), pp. 134-143, September 2016. 
[24] N. G. Cheng, A. Gopinath, L. Wang, K. Iagnemma, and A. E. Hosoi, "Thermally tunable, selfhealing composites for soft robotic applications," Macromol. Mater. Eng., pp. n/a-n/a, 2014.

[25] W. Wang, H. Rodrigue, and S.-H. Ahn, "Deployable soft composite structures," Sci. Rep., vol. 6, p. 20869, 2016.

[26] Y. Chen, J. Sun, Y. Liu, and J. Leng, "Variable stiffness property study on shape memory polymer composite tube," Smart Mater. Struct., vol. 21, no. 9, p. 094021, 2012.

[27] J. Shintake, B. Schubert, S. Rosset, H. Shea, and D. Floreano, "Variable stiffness actuator for soft robotics using dielectric elastomer and low-melting-point alloy," in Proc. IEEE/RSJ Int. Conf. Int. Robot. and Sys., Sept 2015, pp. 1097-1102.

[28] J. Viau, P. Chouinard, J. P. L. Bigue, G. Julio, F. Michaud, and J. S. Plante, "Tendon-driven manipulator actuated by magneto-rheological clutches exhibiting both high-power and soft motion capabilities," IEEE/ASME T-Mech., vol. PP, no. 99, pp. 1-1, 2016.

[29] T. Takeda, Y. Shindo, and F. Narita, "Flexural stiffness variations of woven carbon fiber composite/shape memory polymer hybrid layered beams," J. Comp. Mater., vol. 49, no. 2, pp. 209-216, 2015.

[30] A. Firouzeh and J. Paik, "The design and modeling of a novel resistive stretch sensor with tunable sensitivity," IEEE Sensors Journal, vol. 15, no. 11, pp. 6390-6398, Nov 2015.

[31] A. Firouzeh, Y. Sun, H. Lee, and J. Paik, "Sensor and actuator integrated low-profile robotic origami," in Proc. IEEE/RSJ Int. Conf. Int. Robot. and Sys., Nov 2013, pp. 4937-4944. 